\section{The economic cost of schizophrenia in Ireland}

Dear Editor - Re Letter December 2008 edition of Ir J Psych Med 2008; 25 (4): 157-160. We thank Dr. Dixit for his interest in and positive comments about our study of the economic costs of schizophrenia in Ireland. We wholeheartedly agree that the final figure of more than $€ 460$ million annually may still underestimate the true enormous economic impact schizophrenia has on the taxpayer. However, the goal was to provide an "at least" figure for the cost of the illness, hence the conservative approach to all estimates.

We appreciate Dr Dixit's suggestion to use the Health Research Board reports in estimating the inpatient cost of schizophrenia and apologise if the manuscript was unclear, but this is precisely what we did. We used HIPE $^{1}$ data to estimate the number of inpatient stays with schizophrenia in acute general hospitals only (reference 10 in our study). We used the NPIRS data published by the Health Research Board $^{2}$ to estimate the number of inpatient stays in psychiatric units in general hospitals, in psychiatric hospitals and in private psychiatric hospitals, (reference 9 in our study). The number of admissions with schizophrenia to each type of hospital was multiplied by the average length of stay to give the total days due to schizophrenia. The total cost of inpatient care in any kind of psychiatric hospital or acute unit was $€ 29.9$ million, using the HRB data. The cost of inpatient treatment in general hospitals where schizophrenia was the primary diagnosis was $€ 2.20$ million, using the HIPE data. The total inpatient cost was $€ 32.1$ million. Dr Dixit does not provide the precise methodology for how he derived his final figures so comparison is difficult.

In reference to Dr Dixit's final point regarding including the cost of tribunals in involuntary admissions, the 2001 Mental Health Act became operational in November of 2006 and therefore was not a cost typical of that year. However, we agree entirely with him that the operation of the Act is an added cost and should be included in any evaluation after 2006

While cost of illness studies are useful in estimating the economic burden of a disease, they are limited by the quality of the data available. The only way to improve accuracy is to perform "bottom-up" studies when estimating costs, ie. to take the costs per person diagnosed with the illness in question and to apply them to the total cost. Given the huge cost of schizophrenia to the health service and the taxpayer, it is remarkable that there is a dearth of such studies.

Caragh Behan, Senior Registrar, General Adult Psychiatry, St lta's Portrane

Brendan Kennelly, Department of Economics, National University of Ireland, Galway

Eadbhard O' Callaghan, DETECT, Early Intervention for Psychosis, Dublin \& Dept of Psychiatry, School of Medicine, University College Dublin

References

1. Economic and Social Research Institute 2007 Activity in Acute Public Hospitals in Ireland 2004, Annual report

2. Daly A, Ward M, Moran R, Walsh D. Activities of Irish Psychiatric Units and Hospitals 2006. Health Research Board Annual reports, Dublin 2006)

\section{New executive clinical director posts in Ireland}

Dear Editor - Healthcare has become a complex business and far from a simple exercise, which requires expertise in healthcare management to operate efficiently, especially when resources are limited. Due to increased reliance on economic efficiency, quality of care, consumer involvement, accountability, and scientific methods of service delivery, it is impossible for clinicians to keep themselves away from management issues. The role of consultant is evolving with responsibilities as a clinician, teacher, manager and a team leader.

Some doctors are reluctant to participate in management due to lack of time, manager's reluctance to involve doctors in real management issues, lack of understanding of management culture, and lack of opportunities to get training in healthcare management. Those clinicians say that they are content and rewarded by their clinical work. Even where doctors are keen to get involved in management, they are not sure what is expected of them by the healthcare system, whether it should be, "doctors as managers", "doctors in management", or "doctors and management". This lack of role clarity and a strong inner desire to be involved leaves them frustrated at times. There are few who accept the challenge but want to retain credibility with fellow clinicians by retaining an active clinician's role as well which makes it further difficult for them to fulfil two roles. On the other side it is difficult in our current healthcare structure to make a clinician's role meaningful with realistic involvement in management of healthcare delivery. Ross (1990) states that, "Power without responsibility is dangerous, but responsibility without power is frustrating".

Due to the current competitive environment to get higher specialist training schemes and substantive consultant posts, most doctors do different types of courses ranging from a day course to masters in healthcare management. Those doctors are experiencing difficulties in finding structure which facilitate their involvement in management particularly as a non consultant hospital doctor. In my opinion it is important to facilitate a participative relationship between clinicians and managers which starts from a very early stage as a non consultant hospital doctor and increases as he/she progresses in his/her clinical career which includes management training in their organisational context which will result in a new generation of clinicians as managers.

At present in the Republic of Ireland, we are going through a process of selection or recruitment of executive clinical directors in different specialities and regions without clarifying the role, responsibilities and powers of this new post. It is also decided that if all colleagues unanimously select a consultant as an executive clinical director than there will be no interviews which in my opinion undermines the formal training, experience and management capabilities of the person. In my opinion, there should be a competition for all executive clinical posts, and all executive clinical directors should be given appropriate management training before taking up responsibilities.

Zahid Latif MBBS DCP DPM DMH Dip in CBT MA MRCPsych. 
SpR in Psychiatry, St Davnet's Hospital, Monaghan, Co. Monaghan, Ireland.

\section{Lead $(\mathrm{Pb})$, intellectual functioning and health}

Dear Editor - The latest concerns raised by the Environmental Protection Agency (EPA) over lead ( $\mathrm{Pb}$ ) in water supplied in counties Galway, Donegal, Limerick, North Tipperary and Cork are a worrying, but not a new development in relation to exposure to $\mathrm{Pb}$ in Ireland. In recent years over 10 million toys have been recalled in Europe having found to be contaminated with $\mathrm{Pb}$ or $\mathrm{Pb}$ based paint (the brands involved are market leaders including Reebok, Mattel and Fisher-Price).

Concern about $\mathrm{Pb}$ and the effect on human health is considerable, but may be of particular concern to psychiatrists given its impact on IQ. Although most, if not all adults are aware of the dangers of $\mathrm{Pb}$ poisoning, it is often in a rather broad and non-specific sense. Complacency about this issue is common given that $\mathrm{Pb}$ is usually an unseen and insidious pollutant which many people believe is no longer a real health risk.

\section{Sources of $\mathrm{Pb}$}

$\mathrm{Pb}$ pollution is ubiquitous in the environment and is detectable in all phases of the inert environment such as air, water and soil as well as in most biological systems. It is one of man's most valuable commodities and is mined and processed in over 60 countries. ${ }^{1}$ It has been described as the most important toxic hazard in the development of civilisation and exposure of human populations was considered relatively low until the arrival of the industrial revolution of the 1920s and 1930 s and large-scale mining. ${ }^{2}$ Between the 1930 s and 1970s, the $\mathrm{Pb}$ concentration measured in the Greenland ice sheet was 25 to $\mathbf{5 0}$ times higher than during the Roman times, due in large part to leaded petrol. This was evident in decreasing $\mathrm{Pb}$ concentrations in Greenland snow by a factor of 7.5 over a 20 year period from the late $1960 \mathrm{~s}^{3}$

Although the 1980s and 1990s have seen a large decrease in atmospheric $\mathrm{Pb}$ loads, mainly due to the phasing out of leaded petrol the problem remains. ${ }^{4}$ Most of the millions of tonnes of $\mathrm{Pb}$ burned in petrol in Europe in the 20th century remains in the soil, air and water. Since 1991, all new cars on the roads of Europe are required to run on unleaded petrol. In Ireland legislation recognising the toxicity of $\mathrm{Pb}$ paints was first introduced in 1921 with a ban on $\mathrm{Pb}$ paint for interior use passed in Europe in 1933 and $\mathrm{Pb}$ compounds were finally eliminated from household paints by 1960 .

\section{Exposure}

$\mathrm{Pb}$ is a multimedia exposure problem. The occupational threats of working with $\mathrm{Pb}$ have long been recognised with certain occupations being at particular risk to $\mathrm{Pb}$ exposure such as painters, ironworkers, construction workers, cablesplicers, automobile radiator repair mechanics, firearms instructor, metal shop worker, stained glass artists and battery makers/recyclers. In addition, care may need to be taken in other industrial areas such as paint manufacturing, the crystal glass industries, certain ceramic and craft pottery industries and even elements of the plastic industry (which use $\mathrm{Pb}$ additives), the electronics industry, demolition/salvage work, as well as some elements of the steel industry. Other non-occupational sources of $\mathrm{Pb}$ exposure include $\mathrm{Pb}$ dust (either from paint or mining/ tailings), batteries, ceramics, and certain hobbies such as making stained glass and furniture restoration.

Although $\mathrm{Pb}$ has been banned from paint and petrol for some years now, it remains a constant in our environment. The main avenues of lead uptake among humans are via ingestion and inhalation (and rarely by skin exposure). Many older homes may still have $\mathrm{Pb}$ pipes, and plumbing in newer homes may include $\mathrm{Pb}$ solder. Many people may erroneously assume for example that 'lead free' piping is in fact $\mathrm{Pb}$ free. However counsel from the US EPA indicates that the piping may in fact not be $\mathrm{Pb}$ free but contain $<8 \% \mathrm{~Pb}$.

Other sources of potential contamination include $\mathrm{Pb}$ based paint that is peeling or cracking. Given improvements in legislation that have reduced $\mathrm{Pb}$ in pollutants such as paint and petrol it would be easy to be complacent on this issue. Today, in many parts of the world $\mathrm{Pb}$ toxicity from paint represents a major source of exposure for children. ${ }^{5}$ Children ingest dust and soil contaminated with $\mathrm{Pb}$ from paint, which flakes or chalks as it ages or is disturbed during home maintenance or renovation. ${ }^{6}$

Toxicity

$\mathrm{Pb}$ poisoning is an environmental disease that is the result of human activities. The hazards from $\mathrm{Pb}$ have long been recognised and indications of the consequences have been documented as far back as the 2 nd century BC by Galen and in the 5th century by Hippocrates. Sub-clinical Pb toxicity defined by a whole blood concentration greater than or equal to 10 micrograms per decilitre $(\mu \mathrm{g} / \mathrm{dL})$ affects an estimated one in $\mathbf{2 0}$ children in the United States. Both children and adults are susceptible to health effects from $\mathrm{Pb}$ exposure, but the exposure pathways and effects are somewhat different. Although many body systems can be severely affected by high chronic and acute $\mathrm{Pb}$ exposure, $\mathrm{Pb}$ is a hazard mainly not because of the moderate or low exposure but the chronic exposure that can affect the developing nervous system of young children in more elusive and detrimental ways.

The recent decades have seen a continual reduction in what are deemed 'safe' levels of blood $\mathrm{Pb}$ levels (the intervention level for $\mathrm{BPb}$ in children declined from $60 \mu \mathrm{g} / \mathrm{dL}$ in 1965 to $10 \mu \mathrm{g} / \mathrm{dL}$ in $2000 .^{7}$ Evidence of health impacts at lower and lower levels of exposure continue to emerge in the research literature. ${ }^{8}$ Perhaps most significantly it is important to note that there is no evidence to date that contradicts the suspicion that there may be no threshold below which lead does not exert a toxic effect. Although the current accepted intervention level for $\mathrm{BPb}$ in children is currently $10 \mu \mathrm{g} / \mathrm{dL}$, this level is still disputed with some authors suggesting a reduction to $2 \mu \mathrm{g} / \mathrm{dL}$. ${ }^{9}$

\section{Adverse health and psychological effects}

Although deaths from $\mathrm{Pb}$ pollution are extremely rare, psychologists should be aware of the potential threat to 10 it poses. Recent investigations examining the impact of lead on attention and $10,1^{10}$ including a meta-analysis of seven international population-based longitudinal cohort studies have noted a dose-response relationship between increasing lead and decreasing IQ, indicating the absence of a 'safe' blood lead level. ${ }^{11}$ 